Archive for

Organic Chemistry
Arkivoc 2019, part vi, 0-0

\title{
Efficient synthesis of novel 1-hydroxy-2,4,5-trisubstituted imidazole derivatives via a one-pot, four-component reaction between hydroxylamine, benzonitriles, arylglyoxals and cyclic 1,3-dicarbonyl compounds
}

\author{
Farzaneh Alizadeh-Bami, Mina Salehzadeh, Hossein Mehrabi, * and Reza Ranjbar-Karimi \\ Department of Chemistry, Vali-e-Asr University of Rafsanjan, 77176 Rafsanjan, Iran \\ E-mail: mehrabyh@yahoo.com
}

Received 05-25-2019

Accepted 07-22-2019

Published on line $08-04-2019$

\section{Abstract}

A simple, and efficient procedure for the synthesis of novel 2,5-diaryl-1-hydroxy-1H-imidazol-4-yl derivatives via a one-pot, four-component reaction between hydroxylamine, benzonitriles, arylglyoxals and cyclic 1,3dicarbonyl compounds (Melrum's acid or dimedone) in ethanol under reflux conditions without using bases and catalysts is reported. All the products were obtained in good yields and their structures were established from their spectroscopic data.

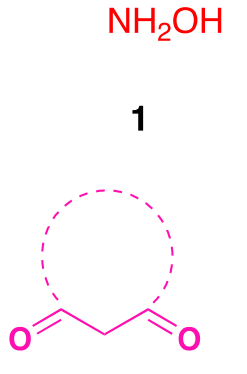

3
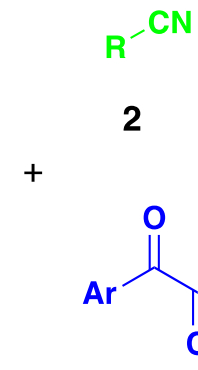

2<smiles>O=CC(=O)Br</smiles>

4

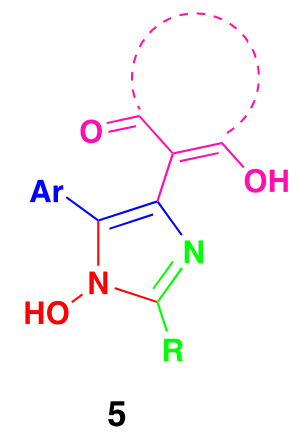

5

Keywords: 1-Hydroxy-2,4,5-trisubstituted imidazoles, hydroxylamine, benzonitriles, arylglyoxals, 1,3dicarbonyl compounds, dimedone, Meldrum's acid 


\section{Introduction}

Hydroxy-imidazole derivatives have attracted great attention in recent years ${ }^{1}$ due to their exhibiting a wide spectrum of biological activities such as the anti-HIV $\mathbf{A},{ }^{2}$ anti-inflammatory $\mathbf{B}^{3}$ antibacterial $\mathbf{C},{ }^{4,5}$ antiprotozoal D, ${ }^{6}$ and antihypertensive $\mathbf{E}$ agents (Figure 1$)^{7}$

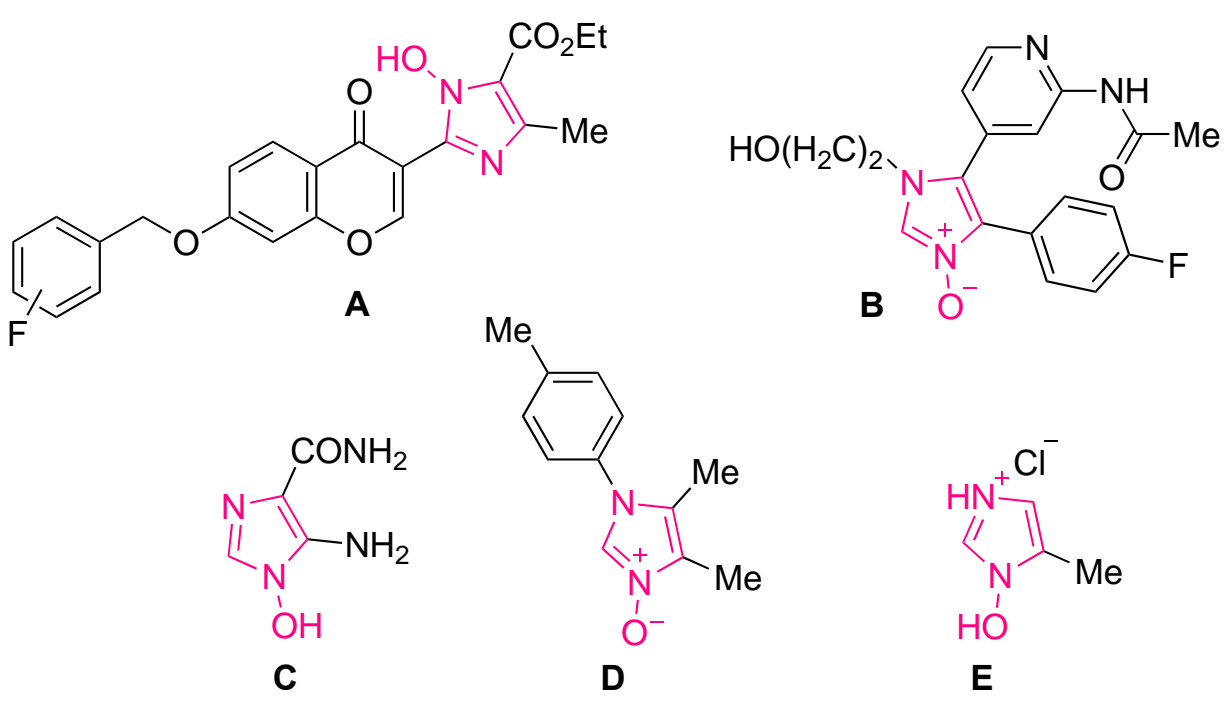

Figure 1. Selected bioactive molecules containing a 1-hydroxy-imidazole moiety.

Some 1-hydroxy-imidazole derivatives, such as metronidazole, ${ }^{8,9}$ are present in drugs for treatment of parasitic diseases and they have applications in biochemistry, dye production, corrosion science, ${ }^{10}$ and as transition metal chelating agents. ${ }^{11}$ Also, 1-hydroxy-imidazoles may be used as insecticides or as plant growth regulators. ${ }^{12,13}$ As a result, studies on efficient syntheses of 1-hydroxy-imidazole derivatives have received much attention.

Over the past years, several successful methods have been reported for the preparation of 1-hydroxy imidazoles and related compounds. The mostly used methods are as follows: (a) condensations of $\alpha$ hydroxyimino ketones with $\mathrm{N}$-methyleneamines; ${ }^{14-16}$ (b) cyclocondensation of $\alpha$-amino oxime derivatives with orthoesters; ${ }^{17}$ (c) reaction of 1,2 -diimines with oxime derivatives; ${ }^{18}$ (d) condensation of $\alpha$-hydroxyimino ketones with various methylidene alkylamines, ${ }^{19}$ (e) three-component reaction of $\alpha$-hydroxyimino ketones with a primary amine and aldehyde derivatives; ${ }^{20}$ (f) via directed lithiation and metal-halogen exchange; ${ }^{7}(\mathrm{~g})$ [3+2] cycloaddition of oximino carbenoids with nitriles; ${ }^{21}$ (h) three-component coupling between $\alpha$ hydroxyimino ketone, aldehydes and ammonium acetate; ${ }^{2}$ (i) four-component reaction of benzil, aromatic aldehydes, an amine, and ammonium acetate; ${ }^{22,23}(\mathrm{j})$ condensation of benzil, an aldehyde, and ammonium acetate in the presence of different catalysts; ${ }^{24,25}$ and $(k)$ one-pot $[2+2+1]$ cycloannulation of 1,3bishet(aryl)monothio-1,3-diketones, $\alpha$-substituted methylamines and sodium nitrite. ${ }^{26}$

However, many of these methods have one or more disadvantages, such as the use of hazardous organic solvents, long reaction times, low yields, tedious work-up procedures, and use of expensive catalysts. With this background in mind and in continuation of our previous work on the development of multicomponent approaches for the synthesis of new heterocycles, ${ }^{27,28}$ we describe a new strategy for the synthesis of 1hydroxy-2,4,5-trisubstituted imidazoles by a one-pot, four-component reaction of a hydroxylamine, a benzonitriles, a cyclic 1,3-dicarbonyl compound, and an arylglyoxal. 


\section{Results and Discussion}

To find the optimized conditions, we studied the synthesis of 5-(5-(4-chlorophenyl)-1-hydroxy-2-phenyl-1Himidazol-4-yl)-6-hydroxy-2,2-dimethyl-4H-1,3-dioxin-4-one $5 \mathrm{a}$ via the four-component reaction of hydroxylamine 1, benzonitrile 2a, Meldrum's acid 3, and 4-chlorophenylglyoxal 4a, with a 2.0:1.0:1.0:1.0 molar ratio, under a variety of conditions (Table 1 ).

Table 1. Optimization of the reaction conditions

\begin{tabular}{|c|c|c|c|}
\hline $\begin{array}{c}\mathrm{NH}_{2} \\
\mathbf{1}\end{array}$ & $2 a$ & $\mathrm{EtOH}$ & $0=$ \\
\hline 3 & $4 a$ & & $5 a$ \\
\hline Entry & Solvent & Temp. $\left({ }^{\circ} \mathrm{C}\right)^{\mathrm{a}}$ & Yield $(\%)^{b}$ \\
\hline 1 & $\mathrm{H}_{2} \mathrm{O}$ & r.t. & Trace \\
\hline 2 & $\mathrm{CH}_{3} \mathrm{CN}$ & r.t. & Trace \\
\hline 3 & DMF & r.t. & Trace \\
\hline 4 & THF & r.t. & Trace \\
\hline 5 & $\mathrm{MeOH}$ & r.t. & Trace \\
\hline 6 & $\mathrm{EtOH}$ & r.t. & Trace \\
\hline 7 & $\mathrm{H}_{2} \mathrm{O}$ & reflux & 10 \\
\hline 8 & $\mathrm{CH}_{3} \mathrm{CN}$ & reflux & 13 \\
\hline 9 & DMF & reflux & 30 \\
\hline 10 & THF & reflux & 15 \\
\hline 11 & $\mathrm{MeOH}$ & reflux & 25 \\
\hline 12 & $\mathrm{EtOH}$ & reflux & 82 \\
\hline 13 & $\mathrm{EtOH}$ & reflux ${ }^{c}$ & 82 \\
\hline
\end{tabular}

${ }^{a}$ Reaction conditions: 1 ( $\left.2.0 \mathrm{mmol}\right), \mathbf{2 a}(1.0 \mathrm{mmol}), \mathbf{3}(1.0 \mathrm{mmol})$, 4a $(1.0 \mathrm{mmol})$, solvent volume $10.0 \mathrm{~mL}$ and reaction time was $4 \mathrm{~h}$.

${ }^{\mathrm{b}}$ Isolated yields.

${ }^{c}$ Reaction time was $24 \mathrm{~h}$.

When the reaction was carried out in a variety of solvents: water, acetonitrile, $\mathrm{N}, \mathrm{N}$-dimethylformamide (DMF), tetrahydrofuran (THF), methanol, and ethanol (Table 1, entries 1-6) at room temperature, only a trace of product was obtained but when the reaction was carried out in water, acetonitrile, DMF, THF, methanol, and ethanol at reflux, the product was obtained in yields of $10 \%, 13 \%, 30 \%, 15 \%, 25 \%$, and $82 \%$ respectively (Table 1, entries 7-12). Extending the reaction time in refluxing ethanol did not improve the yield (Table 1, entry 13). Having optimized conditions in hand, we now explored the scope of this novel transformation for various substituted benzonitriles $\mathbf{2}$, and arylglyoxals $\mathbf{4}$ and the results are summarized in Table 2 . 
As can be seen from Table 2, the nature of the benzonitriles and the arylglyoxal were important. When the benzonitrile, and especially the arylglyoxal, carried electron-withdrawing groups (for example, halide and nitro), higher yields were achieved.

Table 2. Synthesis of 1-hydroxy-2,4,5-trisubstituted imidazole derivatives

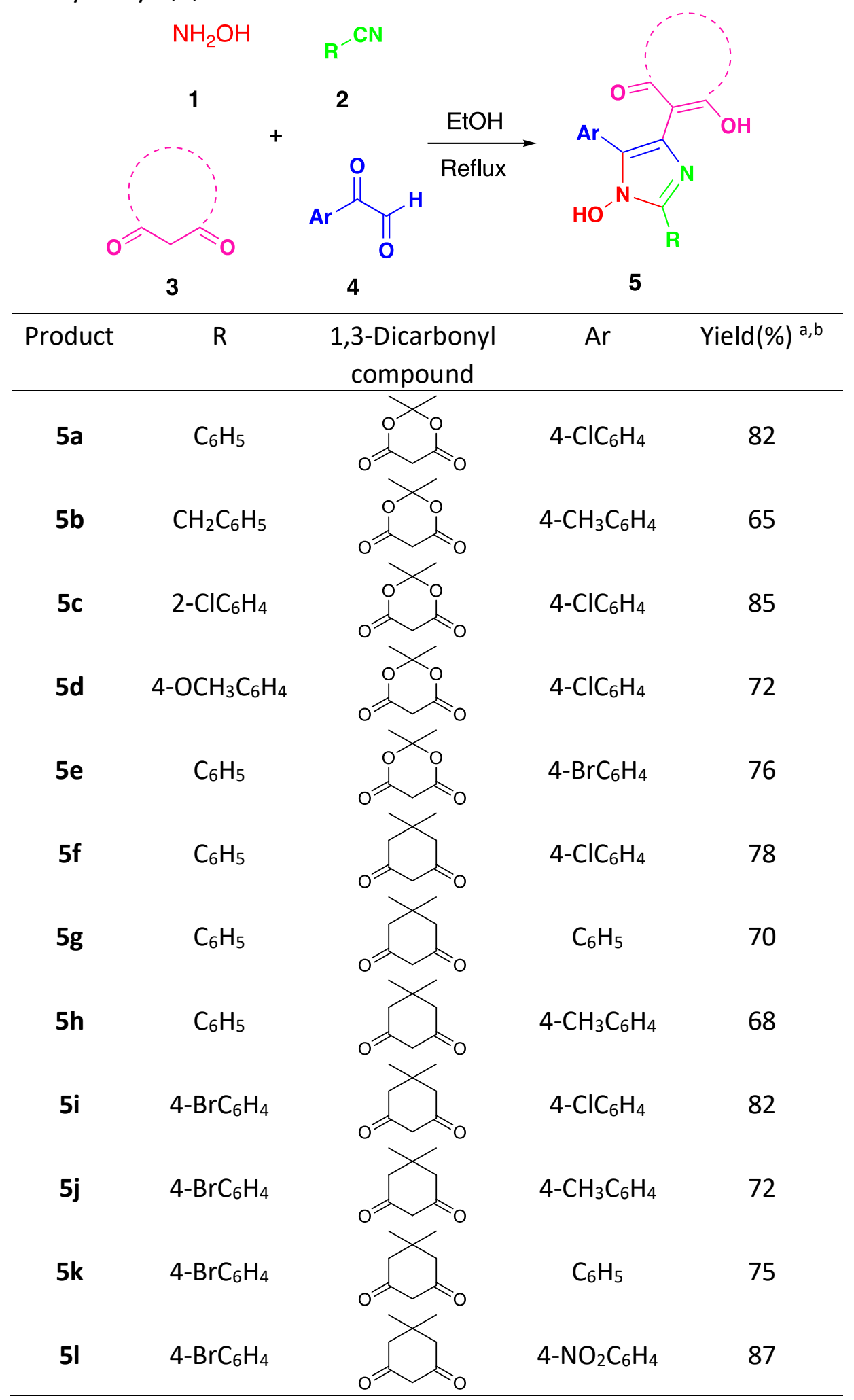

${ }^{a}$ Isolated yields.

${ }^{\mathrm{b}}$ Reaction time was $4 \mathrm{~h}$. 
All the synthesized compounds were unknown and were characterized by ${ }^{1} \mathrm{H}$ and ${ }^{13} \mathrm{C} \mathrm{NMR}, \mathrm{IR}, \mathrm{CHN}$ analysis and melting points. For instance, the ${ }^{1} \mathrm{H}$ NMR spectrum of the compound 5 a consisted of a singlet at $\delta 1.64$ ppm for the methyl groups in the Meldrum's acid unit and a singlet that integrated for one hydrogen was observed at $\delta 2.06 \mathrm{ppm}$ for the hydroxy proton. The aromatic protons resonated in the region $\delta 7.51-8.04$ ppm and a broad singlet that integrated for one hydrogen was observed at $\delta 14.10 \mathrm{ppm}$ for the hydroxy proton. The ${ }^{13} \mathrm{C}$ NMR spectrum of compound 5a exhibited 16 distinct signals in agreement with the proposed structure. In the IR spectrum, the hydroxyl and the carbonyl lactone absorption were observed at 3424 and $1639 \mathrm{~cm}^{-1}$. Partial assignments of these resonances for the other products are given in the Experimental Section.

The possible mechanism for the synthesis of 1-hydroxy-2,4,5-trisubstituted imidazole derivatives $\mathbf{5}$ is illustrated in Scheme 1. Firstly, intermediate $\mathbf{A}$ is formed by the addition of the hydroxylamine $\mathbf{1}$ to the benzonitrile 2. Also, intermediate $\mathbf{B}$ is formed by means of a Knoevenagel condensation between the arylglyoxal 3 and 1,3-dicarbonyl compound 4, Meldrum's acid or dimedone. Then, Michael addition of amidoxime $\mathbf{A}$ (as Michael donor) to $\alpha, \beta$-unsaturated $\gamma$-dicarbonyl species $\mathbf{B}$ (as Michael acceptor) provides intermediate $\mathbf{C}$, which subsequently undergoes intramolecular nucleophilic addition to form intermediate $\mathbf{D}$. In the last step, 1-hydroxy-2,4,5-trisubstituted imidazole derivative $\mathbf{5}$ is formed by elimination of $\mathrm{H}_{2} \mathrm{O}$ and intramolecular $\mathrm{H}$-shift.
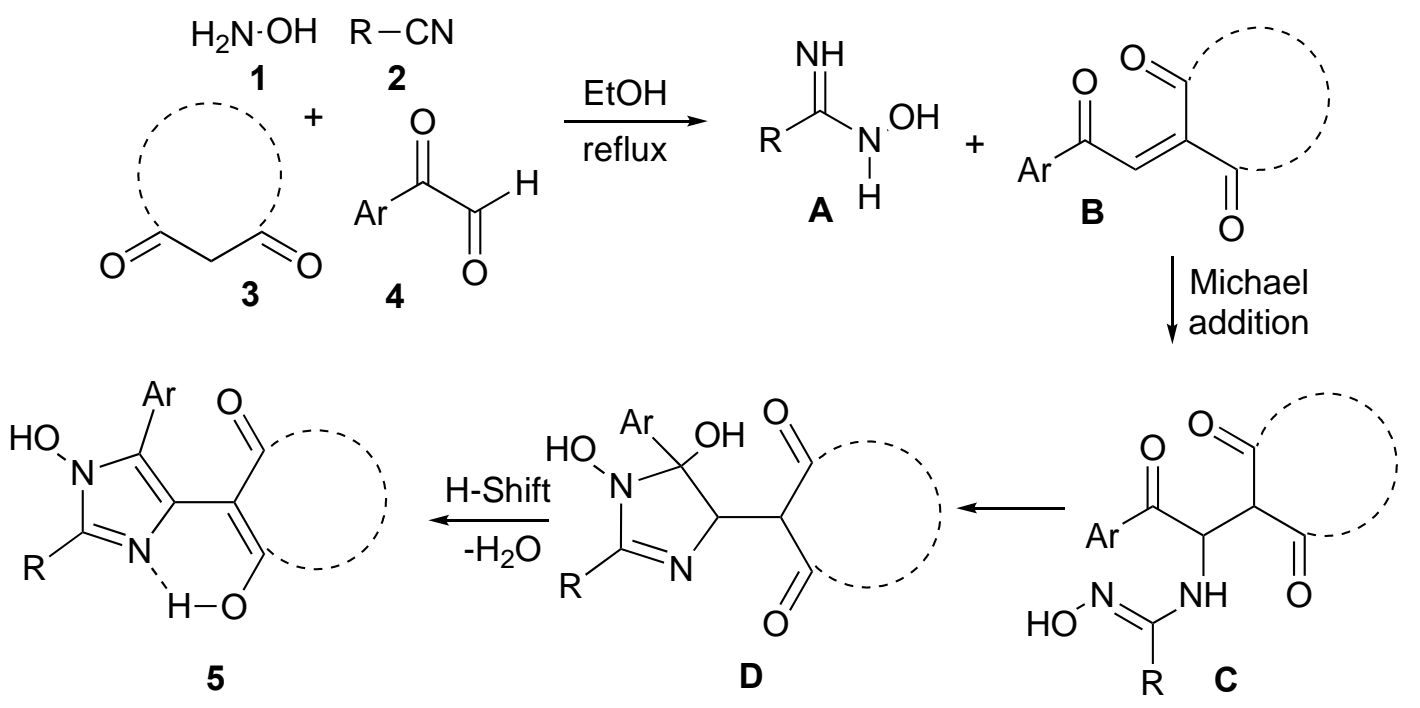

Scheme 1. Proposed mechanism of the reaction.

\section{Conclusions}

We have successfully developed a novel and four-component reaction for synthesis of 1-hydroxy-2,4,5trisubstituted imidazoles via hydroxylamine, benzonitriles, arylglyoxal and Meldrum's acid or dimedone in ethanol under reflux conditions. This efficient strategy has the advantages of catalyst-free, easy work-up, high yields, short reaction time, and an environmentally benign procedure. 


\section{Experimental Section}

General. All chemicals were purchased from Aldrich and Merck with high-grade quality, and used without any purification. All melting points were obtained by Barnstead Electrothermal 9200 apparatus and are uncorrected. The reactions were monitored by TLC and all yields refer to isolated products. NMR spectra were obtained on a Varian $500 \mathrm{MHz}$ spectrometer $\left({ }^{1} \mathrm{H} \mathrm{NMR}\right.$ at $500 \mathrm{MHz},{ }^{13} \mathrm{C}$ NMR at $\left.125 \mathrm{MHz}\right)$ in DMSO-d $d_{6}$ using TMS as an internal standard. Infrared spectra were recorded on a Bruker FT-IR Equinax-55 spectrophotometer in $\mathrm{KBr}$ with absorption in $\mathrm{cm}^{-1}$. Elemental analyses were performed using a Carlo Erba EA 1108 instrument. All products were characterized by their spectroscopic and physical data.

\section{General procedure for the synthesis of 1-hydroxy-2,4,5-trisubstituted imidazole derivatives.}

A mixture of hydroxylamine (1) $(2.0 \mathrm{mmol})$ and benzonitrile $(2)(1.0 \mathrm{mmol})$ was stirred in EtOH $(10 \mathrm{~mL})$ at reflux for $3 \mathrm{~h}$ to give the amidoxime. Then, Meldrum's acid (3) $(1.0 \mathrm{mmol})$ and an arylglyoxal (4) (1.0 mmol) were added, and the resulting mixture stirred at reflux for $1 \mathrm{~h}$. After completion of the reaction, determined by TLC analysis, the solvent was removed under reduced pressure, and the resulting crude product was purified by recrystallization from EtOH to give the pure compounds 5a-I (65-87\%).

\section{5-(5-(4-Chlorophenyl)-1-hydroxy-2-phenyl-1H-imidazol-4-yl)-6-hydroxy-2,2-dimethyl-4H-1,3-dioxin-4-one}

(5a). $\mathrm{mp} 213-215^{\circ} \mathrm{C}$. IR v/cm ${ }^{-1}$ (KBr): 3424, 2991, 1639. ${ }^{1} \mathrm{H}$ NMR (500 MHz, DMSO-d 6 ): $\delta 1.64\left(\mathrm{~s}, 6 \mathrm{H}, 2 \mathrm{CH}_{3}\right)$, 2.06 (s, 1H, OH), 7.51 (d, J 10.0 Hz, 2H, ArH), 7.55 (s, 2H, ArH), 7.56 (d, J 5.0 Hz, 2H, ArH), 7.63 (s, 1H, ArH), 8.04 $(\mathrm{m}, 2 \mathrm{H}, \mathrm{ArH}), 14.10(\mathrm{~s}, 1 \mathrm{H}, \mathrm{OH}) \mathrm{ppm} .{ }^{13} \mathrm{C}$ NMR $\left(125 \mathrm{MHz}, \mathrm{DMSO}-d_{6}\right): \delta 26.2,102.2,122.3,125.4,126.5,128.5$, $129.1,129.3,129.8,131.5,131.5,131.8,135.9,161.8,165.3,196.5$ ppm. Anal. Calcd for $\mathrm{C}_{21} \mathrm{H}_{17} \mathrm{ClN}_{2} \mathrm{O}_{5}(412.83)$ : C, 61.10; H, 4.15; N, 6.79. Found: C, 61.24; H, 4.17; N, 6.75\%.

5-(2-Benzyl-1-hydroxy-5-(p-tolyl)-1H-imidazol-4-yl)-6-hydroxy-2,2-dimethyl-4H-1,3-dioxin-4-one (5b). mp 208-211 ${ }^{\circ} \mathrm{C}$. IR v/cm ${ }^{-1}$ (KBr): 3432, 2998, 1667; ${ }^{1} \mathrm{H}$ NMR $\left(500 \mathrm{MHz}, \mathrm{DMSO}-d_{6}\right): \delta 1.22\left(\mathrm{~s}, 6 \mathrm{H}, 2 \mathrm{CH}_{3}\right), 1.58$ (s, 3H, $\left.\mathrm{CH}_{3}\right), 2.07(\mathrm{~s}, 1 \mathrm{H}, \mathrm{OH}), 3.76\left(\mathrm{~s}, 2 \mathrm{H}, \mathrm{CH}_{2}\right), 6.67(\mathrm{~d}, J 10.0 \mathrm{~Hz}, 2 \mathrm{H}, \mathrm{ArH}), 6.78(\mathrm{~d}, J 10.0 \mathrm{~Hz}, 1 \mathrm{H}, \operatorname{ArH}), 6.82(\mathrm{~s}, 2 \mathrm{H}$, ArH), 6.84 (d, J $7.9 \mathrm{~Hz}, 2 \mathrm{H}, \mathrm{ArH}), 6.92$ (d, $2 \mathrm{H}, J 7.9 \mathrm{~Hz}, \mathrm{ArH}), 13.31$ (s, 1H, OH) ppm. ${ }^{13} \mathrm{C}$ NMR (125 MHz, DMSO$\left.d_{6}\right): \delta 20.8,25.6,28.9,62.6,101.7,123.2,123.7,125.8,126.1,127.1,128.3,128.5,128.6,133.6,136.1,137.6$, 165.1, 191.2 ppm. Anal. Calcd for $\mathrm{C}_{23} \mathrm{H}_{22} \mathrm{~N}_{2} \mathrm{O}_{5}$ (406.44): $\mathrm{C}, 67.97 ; \mathrm{H}, 5.46 ; \mathrm{N}, 6.89$. Found: $\mathrm{C}, 67.19 ; \mathrm{H}, 5.50 ; \mathrm{N}$, $6.91 \%$.

5-(2-(2-Chlorophenyl)-5-(4-chlorophenyl)-1-hydroxy-1H-imidazol-4-yl)-6-hydroxy-2,2-dimethyl-4H-1,3dioxin-4-one (5c). $\mathrm{mp}$ 204-206 ${ }^{\circ} \mathrm{C}$. IR v/cm ${ }^{-1}$ (KBr): 3420, 2987, 1609; ${ }^{1} \mathrm{H}$ NMR (500 MHz, DMSO-d $)$ ): $\delta 1.05$ (s, $1 \mathrm{H}, \mathrm{OH}), 1.65\left(\mathrm{~s}, 6 \mathrm{H}, 2 \mathrm{CH}_{3}\right), 7.55(\mathrm{~d}, J 5.0 \mathrm{~Hz}, 2 \mathrm{H}, \mathrm{ArH}), 7.64(\mathrm{~d}, J 5.0 \mathrm{~Hz}, 2 \mathrm{H}, \mathrm{ArH}), 7.71(\mathrm{t}, 2 \mathrm{H}, \operatorname{ArH}), 7.78(\mathrm{~d}, J 6.9$ $\mathrm{Hz}, 1 \mathrm{H}, \mathrm{ArH}), 7.87$ (d, J $7.7 \mathrm{~Hz}, 1 \mathrm{H}, \mathrm{ArH}), 13.85$ (s, 1H, OH) ppm. ${ }^{13} \mathrm{C}$ NMR (125 MHz, DMSO-d $): \delta$ 26.3, 69.2, 102.0, 111.8, 115.6, 122.4, 128.1, 128.4, 128.8, 129.0, 130.5, 133.6, 134.0, 134.7, 135.1, 144.2, 164.8, 214.0 ppm. Anal. Calcd for $\mathrm{C}_{21} \mathrm{H}_{16} \mathrm{Cl}_{2} \mathrm{~N}_{2} \mathrm{O}_{5}$ (447.27): $\mathrm{C}, 56.39 ; \mathrm{H}, 3.61 ; \mathrm{N}, 6.26 \%$.

5-(5-(4-Chlorophenyl)-1-hydroxy-2-(4-methoxyphenyl)-1H-imidazol-4-yl)-6-hydroxy-2,2-dimethyl-4H-1,3dioxin-4-one (5d). $\mathrm{mp} 203-205{ }^{\circ} \mathrm{C}$. IR v/cm ${ }^{-1}$ (KBr): 3422, 2938, 1610. ${ }^{1} \mathrm{H}$ NMR (500 MHz, DMSO-d $): \delta 1.61$ (s, $\left.6 \mathrm{H}, 2 \mathrm{CH}_{3}\right), 2.08(\mathrm{~s}, 1 \mathrm{H}, \mathrm{OH}), 3.86\left(\mathrm{~s}, 3 \mathrm{H}, \mathrm{CH}_{3}\right), 7.20(\mathrm{~d}, J 9.0 \mathrm{~Hz}, 2 \mathrm{H}, \mathrm{ArH}), 7.54(\mathrm{~d}, J 8.6 \mathrm{~Hz}, 2 \mathrm{H}, \mathrm{ArH}), 7.69$ (d, J 8.6 $\mathrm{Hz}, 2 \mathrm{H}, \mathrm{ArH}), 8.04(\mathrm{~d}, J 9.0 \mathrm{~Hz}, 2 \mathrm{H}, \mathrm{ArH}), 13.51(\mathrm{~s}, 1 \mathrm{H}, \mathrm{OH}) \mathrm{ppm} .{ }^{13} \mathrm{C} \mathrm{NMR}\left(125 \mathrm{MHz}, \mathrm{DMSO}-d_{6}\right): \delta 26.3,56.0$, $68.1,101.7,111.3,115.0,126.6,128.4,128.6,128.8,129.2,130.4,133.2,136.9,141.1,164.9,217.0$ ppm. Anal. Calcd for $\mathrm{C}_{22} \mathrm{H}_{19} \mathrm{ClN}_{2} \mathrm{O}_{6}$ (442.85): $\mathrm{C}, 59.67 ; \mathrm{H}, 4.32 ; \mathrm{N}, 6.33$. Found: $\mathrm{C}, 59.79 ; \mathrm{H}, 4.33 ; \mathrm{N}, 6.28 \%$.

5-(5-(4-Bromophenyl)-1-hydroxy-2-phenyl-1H-imidazol-4-yl)-6-hydroxy-2,2-dimethyl-4H-1,3-dioxin-4-one (5e). $\mathrm{mp} 218-220^{\circ} \mathrm{C}$. IR v/cm ${ }^{-1}$ (KBr): 3426, 2986, 1639. ${ }^{1} \mathrm{H} \mathrm{NMR}\left(500 \mathrm{MHz}, \mathrm{DMSO}-d_{6}\right): \delta 1.63\left(\mathrm{~s}, 6 \mathrm{H}, 2 \mathrm{CH}_{3}\right)$, 
2.08 (s, 1H, OH), 7.55 (d, J $5.0 \mathrm{~Hz}, 2 \mathrm{H}, \mathrm{ArH}), 7.64$ (d, J $5.0 \mathrm{~Hz}, 2 \mathrm{H}, \mathrm{ArH}), 7.68(\mathrm{~s}, 1 \mathrm{H}, \mathrm{ArH}), 7.70(\mathrm{~d}, J 10.0 \mathrm{~Hz}, 2 \mathrm{H}$, $\operatorname{ArH}), 8.07$ (d, J 10.0 Hz, 2H, ArH), 13.75 (s, 1H, OH) ppm. ${ }^{13} \mathrm{C}$ NMR (125 MHz, DMSO-d $): \delta$ 26.3, 78.7, 101.8, $127.4,128.7,128.9,129.3,129.5,131.4,131.7,132.8,133.9,136.7,140.7,164.9,187.4$ ppm. Anal. Calcd for $\mathrm{C}_{21} \mathrm{H}_{17} \mathrm{BrN}_{2} \mathrm{O}_{5}$ (457.28): C, 55.16; $\mathrm{H}, 3.75 ; \mathrm{N}, 6.13$. Found: $\mathrm{C}, 55.24 ; \mathrm{H}, 3.77 ; \mathrm{N}, 6.08 \%$.

2-(5-(4-Chlorophenyl)-1-hydroxy-2-phenyl-1H-imidazol-4-yl)-3-hydroxy-5,5-dimethylcyclohex-2-en-1-one

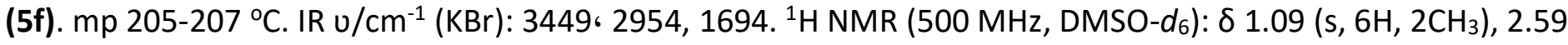
(s, $1 \mathrm{H}, \mathrm{OH}), 3.17\left(\mathrm{~s}, 2 \mathrm{H}, \mathrm{CH}_{2}\right), 3.32\left(\mathrm{~d}, 2 \mathrm{H}, \mathrm{CH}_{2}\right), 7.55$ (d, J $\left.5.0 \mathrm{~Hz}, 2 \mathrm{H}, \mathrm{ArH}\right), 7.57(\mathrm{~s}, 1 \mathrm{H}, \mathrm{ArH}), 7.61(\mathrm{~d}, J 10.0 \mathrm{~Hz}$, $2 \mathrm{H}, \mathrm{ArH}), 7.81$ (d, J $5.0 \mathrm{~Hz}, 2 \mathrm{H}, \mathrm{ArH}), 8.43(\mathrm{~d}, J 10.0 \mathrm{~Hz}, 2 \mathrm{H}, \mathrm{ArH}), 14.01$ (s, 1H, OH) ppm. ${ }^{13} \mathrm{C} \mathrm{NMR}(125 \mathrm{MHz}$, DMSO-d $)_{6}$ : $\delta$ 28.1, 32.9, 45.7, 51.4, 120.7, 129.2, 129.4, 129.6, 131.3, 132.9, 133.5, 136.0, 139.5, 164.4, 165.5, 173.5, 192.2, 196.7 ppm. Anal. Calcd for $\mathrm{C}_{23} \mathrm{H}_{21} \mathrm{ClN}_{2} \mathrm{O}_{3}$ (408.88): C, 67.56; H, 5.18; N, 6.58. Found: $\mathrm{C}, 67.72 ; \mathrm{H}$, $5.23 ; \mathrm{N}, 6.56 \%$.

3-Hydroxy-2-(1-hydroxy-2,5-diphenyl-1H-imidazol-4-yl)-5,5-dimethylcyclohex-2-en-1-one (5g). mp 188-190 ${ }^{\circ} \mathrm{C} . \mathrm{IR} \mathrm{v} / \mathrm{cm}^{-1}(\mathrm{KBr}): 3362,2952,1690 .{ }^{1} \mathrm{H}$ NMR (500 MHz, DMSO-d $\left.)_{6}\right): \delta 1.09\left(\mathrm{~s}, 6 \mathrm{H}, 2 \mathrm{CH}_{3}\right), 2.49(\mathrm{~s}, 1 \mathrm{H}, \mathrm{OH}), 2.59$ $\left(\mathrm{s}, 2 \mathrm{H}, \mathrm{CH}_{2}\right), 3.18\left(\mathrm{~s}, 2 \mathrm{H}, \mathrm{CH}_{2}\right), 7.53(\mathrm{~d}, J 10.0 \mathrm{~Hz}, 2 \mathrm{H}, \mathrm{ArH}), 7.54(\mathrm{~d}, J 10.0 \mathrm{~Hz}, 2 \mathrm{H}, \mathrm{ArH}), 7.59(\mathrm{~m}, 1 \mathrm{H}, \mathrm{ArH}), 7.67$ $(\mathrm{m}, 1 \mathrm{H}, \mathrm{ArH}), 7.78(\mathrm{~d}, J 5.0 \mathrm{~Hz}, 2 \mathrm{H}, \mathrm{ArH}), 8.41(\mathrm{~d}, J 10.0 \mathrm{~Hz}, 2 \mathrm{H}, \mathrm{ArH}), 14.10(\mathrm{~s}, 1 \mathrm{H}, \mathrm{OH}) \mathrm{ppm} .{ }^{13} \mathrm{C} \mathrm{NMR}(125 \mathrm{MHz}$, DMSO-d $)_{\text {): } \delta} 28.1,32.8,45.7,51.5,120.7,129.1,129.2,129.4,129.5,132.8,134.5,134.8,136.1,164.9,165.5$, 173.4, 193.2, 196.6 ppm. Anal. Calcd for $\mathrm{C}_{23} \mathrm{H}_{22} \mathrm{~N}_{2} \mathrm{O}_{3}$ (374.44): C, 73.78; $\mathrm{H}, 5.92 ; \mathrm{N}, 7.48$. Found: $\mathrm{C}, 73.66 ; \mathrm{H}$, $5.91 ; \mathrm{N}, 7.44 \%$.

3-Hydroxy-2-(1-hydroxy-2-phenyl-5-(p-tolyl)-1H-imidazol-4-yl)-5,5-dimethylcyclohex-2-en-1-one (5h). mp 150-153 ${ }^{\circ} \mathrm{C}$. IR v/cm ${ }^{-1}$ (KBr): 3450, 2958, 1682. ${ }^{1} \mathrm{H}$ NMR (500 MHz, DMSO-d $): \delta 1.17\left(\mathrm{~s}, 6 \mathrm{H}, 2 \mathrm{CH}_{3}\right), 2.05$ (s, $1 \mathrm{H}$, $\mathrm{OH}), 2.42\left(\mathrm{~s}, 3 \mathrm{H}, \mathrm{CH}_{3}\right), 2.55\left(\mathrm{~s}, 2 \mathrm{H}, \mathrm{CH}_{2}\right), 3.14\left(\mathrm{~s}, 2 \mathrm{H}, \mathrm{CH}_{2}\right), 7.26(\mathrm{~s}, 2 \mathrm{H}, \mathrm{ArH}), 7.50(\mathrm{~m}, 2 \mathrm{H}, \mathrm{ArH}), 7.52(\mathrm{~d}, J 8.3 \mathrm{~Hz}$, $1 \mathrm{H}, \mathrm{ArH}), 7.72$ (d, J $7.0 \mathrm{~Hz}, 2 \mathrm{H}, \mathrm{ArH}), 8.56$ (d, J $7.0 \mathrm{~Hz}, 2 \mathrm{H}, \mathrm{ArH}), 13.81$ (s, 1H, OH) ppm. ${ }^{13} \mathrm{C} \mathrm{NMR}(125 \mathrm{MHz}$, DMSO- $\left.d_{6}\right): \delta 21.8,28.3,32.7,46.3,52.0,117.2,120.4,128.6,129.4,129.4,132.1,136.1,138.9,144.7,148.5$, 165.6, 172.0, 195.5, 197.5 ppm. Anal. Calcd for $\mathrm{C}_{24} \mathrm{H}_{24} \mathrm{~N}_{2} \mathrm{O}_{3}$ (388.47): C, 74.21; $\mathrm{H}, 6.23 ; \mathrm{N}, 7.21$. Found: C, 74.37; $\mathrm{H}, 6.29 ; \mathrm{N}, 7.18 \%$.

2-(2-(4-Bromophenyl)-5-(4-chlorophenyl)-1-hydroxy-1H-imidazol-4-yl)-3-hydroxy-5,5-dimethylcyclohex-2-

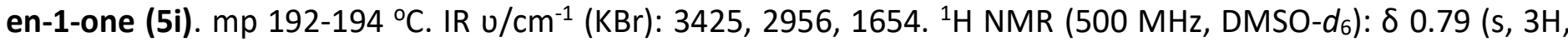
$\left.\mathrm{CH}_{3}\right), 0.81\left(\mathrm{~s}, 3 \mathrm{H}, \mathrm{CH}_{3}\right), 2.11\left(\mathrm{~s}, 2 \mathrm{H}, \mathrm{CH}_{2}\right), 2.31\left(\mathrm{~s}, 2 \mathrm{H}, \mathrm{CH}_{2}\right), 2.66(\mathrm{~s}, 1 \mathrm{H}, \mathrm{OH}), 7.03(\mathrm{~d}, J 8.7 \mathrm{~Hz}, 2 \mathrm{H}, \mathrm{ArH}), 7.11(\mathrm{~d}, J$ $8.6 \mathrm{~Hz}, 2 \mathrm{H}, \mathrm{ArH}), 7.42$ (d, J $8.7 \mathrm{~Hz}, 2 \mathrm{H}, \mathrm{ArH}), 7.59$ (d, J $8.6 \mathrm{~Hz}, 2 \mathrm{H}, \mathrm{ArH}), 13.05(\mathrm{~s}, 1 \mathrm{H}, \mathrm{OH}) \mathrm{ppm} .{ }^{13} \mathrm{C}$ NMR (125 $\left.\mathrm{MHz}, \mathrm{DMSO}-d_{6}\right): \delta 27.9,28.0,30.3,51.7,51.9,104.0,109.6,125.1,126.0,127.7,128.3,129.2,132.2,134.1$, $136.7,156.3,179.0,199.2,199.9 \mathrm{ppm}$.

\section{2-(2-(4-Bromophenyl)-1-hydroxy-5-(p-tolyl)-1H-imidazol-4-yl)-3-hydroxy-5,5-dimethylcyclohex-2-en-1-one}

(5j). $\mathrm{mp} 193-195{ }^{\circ} \mathrm{C}$. IR v/cm ${ }^{-1}(\mathrm{KBr}): 3432,2954,1654 .{ }^{1} \mathrm{H} \mathrm{NMR}\left(500 \mathrm{MHz}, \mathrm{DMSO}-d_{6}\right): \delta 0.84\left(\mathrm{~s}, 3 \mathrm{H}, \mathrm{CH}_{3}\right), 0.87$ $\left(\mathrm{s}, 3 \mathrm{H}, \mathrm{CH}_{3}\right), 2.12\left(\mathrm{~s}, 3 \mathrm{H}, \mathrm{CH}_{3}\right), 2.15\left(\mathrm{~s}, 2 \mathrm{H}, \mathrm{CH}_{2}\right), 2.37\left(\mathrm{~s}, 2 \mathrm{H}, \mathrm{CH}_{2}\right), 2.54(\mathrm{~s}, 1 \mathrm{H}, \mathrm{OH}), 6.92(\mathrm{~d}, J 8.0 \mathrm{~Hz}, 2 \mathrm{H}, \mathrm{ArH})$, 7.10 (d, J $8.4 \mathrm{~Hz}, 2 \mathrm{H}, \mathrm{ArH}), 7.48$ (d, J $8.0 \mathrm{~Hz}, 2 \mathrm{H}, \mathrm{ArH}), 7.66$ (d, J $8.4 \mathrm{~Hz}, 2 \mathrm{H}, \mathrm{ArH}), 13.15$ (s, $1 \mathrm{H}, \mathrm{OH}) \mathrm{ppm} .{ }^{13} \mathrm{C} \mathrm{NMR}$ (125 MHz, DMSO- $\left.d_{6}\right): \delta$ 21.0, 28.0, 28.1, 30.3, 51.8, 51.9, 104.6, 109.7, 124.3, 125.4, 127.5, 128.9, 129.1, 132.2, 134.9, 138.3, 155.8, 180.1, 199.1, 200.0 ppm. Anal. Calcd for $\mathrm{C}_{24} \mathrm{H}_{23} \mathrm{BrN}_{2} \mathrm{O}_{3}$ (467.36): C, 61.68; $\mathrm{H}, 4.96 ; \mathrm{N}, 5.99$. Found: C, 61.70; H, 4.99; N, 6.04\%.

\section{2-(2-(4-Bromophenyl)-1-hydroxy-5-phenyl-1H-imidazol-4-yl)-3-hydroxy-5,5-dimethylcyclohex-2-en-1-one}

(5k). mp 197-199 ${ }^{\circ} \mathrm{C}$. IR v/cm ${ }^{-1}$ (KBr): 3433, 2924, 1655. ${ }^{1} \mathrm{H}$ NMR (500 MHz, DMSO-d $)$ : $\delta 0.86$ (s, 3H, $\left.\mathrm{CH}_{3}\right), 0.90$ $\left(\mathrm{s}, 3 \mathrm{H}, \mathrm{CH}_{3}\right), 2.18\left(\mathrm{~s}, 2 \mathrm{H}, \mathrm{CH}_{2}\right), 2.40(\mathrm{~s}, 1 \mathrm{H}, \mathrm{OH}), 2.50\left(\mathrm{~s}, 2 \mathrm{H}, \mathrm{CH}_{2}\right), 7.15(\mathrm{~d}, J 5.0 \mathrm{~Hz}, 2 \mathrm{H}, \mathrm{ArH}), 7.25(\mathrm{~s}, 2 \mathrm{H}, \mathrm{ArH})$, 7.52 (d, J $5.0 \mathrm{~Hz}, 2 \mathrm{H}, \mathrm{ArH}), 7.55$ (s, 1H, ArH), 7.69 (d, J $7.1 \mathrm{~Hz}, 2 \mathrm{H}, \mathrm{ArH}), 13.18(\mathrm{~s}, 1 \mathrm{H}, \mathrm{OH}) \mathrm{ppm} .{ }^{13} \mathrm{C}$ NMR (125 $\left.\mathrm{MHz}, \mathrm{DMSO}-d_{6}\right): \delta 28.0,28.0,30.4,51.8,51.9,104.7,117.0,124.4,127.7,128.2,128.5,129.2,130.7,132.2$, $132.2,138.7,156.0,180.0,199.1 \mathrm{ppm}$. 
2-(2-(4-Bromophenyl)-1-hydroxy-5-(4-nitrophenyl)-1H-imidazol-4-yl)-3-hydroxy-5,5-dimethyl cyclohex-2-en-

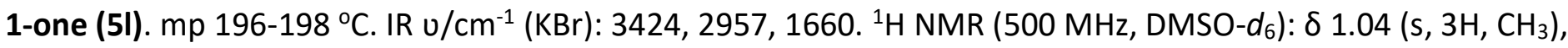
$1.07\left(\mathrm{~s}, 3 \mathrm{H}, \mathrm{CH}_{3}\right), 1.18(\mathrm{~s}, 1 \mathrm{H}, \mathrm{OH}), 2.35\left(\mathrm{~s}, 2 \mathrm{H}, \mathrm{CH}_{2}\right), 2.58\left(\mathrm{~s}, 2 \mathrm{H}, \mathrm{CH}_{2}\right), 7.61(\mathrm{~m}, 2 \mathrm{H}, \mathrm{ArH}), 7.68(\mathrm{~d}, J 8.1 \mathrm{~Hz}, 2 \mathrm{H}$, $\operatorname{ArH}), 7.83(\mathrm{~d}, J 8.1 \mathrm{~Hz}, 2 \mathrm{H}, \mathrm{ArH}), 8.18(\mathrm{~d}, J 8.3 \mathrm{~Hz}, 2 \mathrm{H}, \mathrm{ArH}), 13.32(\mathrm{~s}, 1 \mathrm{H}, \mathrm{OH}) \mathrm{ppm} .{ }^{13} \mathrm{C}$ NMR $(125 \mathrm{MHz}, \mathrm{DMSO}-$ $\left.d_{6}\right): \delta 28.1,28.3,30.6,51.9,52.1,104.0,109.8,123.7,124.9,125.7,128.4,129.3,132.5,145.1,148.0,157.3$, 178.5, 199.5, 200.4 ppm. Anal. Calcd for $\mathrm{C}_{23} \mathrm{H}_{20} \mathrm{BrN}_{3} \mathrm{O}_{5}$ (498.33): C, 55.44; H, 4.05; N, 8.43. Found: C, 55.27; $\mathrm{H}$, $4.01 ; \mathrm{N}, 8.37 \%$.

\section{Supplementary Material}

The Experimental Section and the ${ }^{1} \mathrm{H}$ and ${ }^{13} \mathrm{C}$ NMR spectra for compounds $5 \mathrm{a}-\mathrm{I}$ can be found using the link "Supplementary Material" in the journal issue contents page.

\section{References}

1. Mloston, G.; Jasinski, M.; Linden, A.; Heimgartner, H. Helv. Chim. Acta 2006, 89, 1304. https://doi.org/10.1002/hlca.200690129

2. Nikitina, P. A.; Tkach, I. I.; Knyazhanskaya, E. S.; Gottikh, M. B.; Perevalov, V. P. Pharm. Chem. J. 2016, 50, 513.

https://doi.org/10.1007/s11094-016-1479-x

3. Laufer, S. A.; Zimmermann, W.; Ruff, K. J. J. Med. Chem. 2004, 47, 6311. https://doi.org/10.1021/jm0496584

4. Harnden, M. R.; Jennings, L. J.; Mckie, C. M.; Parkin, A. Synthesis 1990, 10, 893. https://doi.org/10.1055/s-1990-27045

5. Khan, M. S.; Siddiqui, S. A.; Siddiqui, M. S.; Goswami, U.; Srinivasan, K. V.; Khan, M. I. Chem. Biol. Drug. Des. 2008, 72, 197.

https://doi.org/10.1111/j.1747-0285.2008.00691.x

6. Aguirre, G.; Boiani, M.; Cerecetto, H.; Gerpe, A.; Gonzalez, M.; Sainz, Y. F.; Denicola, A.; De Ocariz, C. O.; Nogal, J. J.; Montero, D.; Escario, J. A. Arch. Pharm. Pharm. Med. Chem. 2004, 337, 259.

https://doi.org/10.1002/ardp.200300840

7. Eriksen, B. L.; Vedso, P.; Begtrup, M. J. Org. Chem. 2001, 66, 8344.

https://doi.org/10.1021/jo001554n

8. Boiani, M.; Gonzalez, M. Mini-Rev. Med. Chem. 2005, 5, 409.

https://doi.org/10.2174/1389557053544047

9. Cerecetto, H.; Gonzalez, M. Curr. Top. Med. Chem. 2002, 2, 1187. https://doi.org/10.2174/1568026023393066

10. Davila-Jimenez, M. M.; Elizalde-Gonzalez, M. P.; Garcia-Diaz, S. J. Liq. Chromatogr. R. T. 1998, $21,1629$. https://doi.org/10.1080/10826079808001249

11. Lukomska-Rogala, M.; Rybarczyk-Pirek, A. J.; Ejsmont, K.; Jasinski, M.; Palusiak, M. Struct. Chem. 2017, 28, 1229.

https://doi.org/10.1007/s11224-017-0935-x 
12. Begtrup, M. Adv. Heterocycl. Chem. 2012, 106, 1. https://doi.org/10.1016/B978-0-12-396531-8.00001-8

13. Rybarczyk-Pirek, A. J.; Lukomska, M.; Ejsmont, K.; Jasinski, M.; Palusiak, M. Struct. Chem. 2014, $25,979$. https://doi.org/10.1007/s11224-014-0404-8

14. Mloston, G.; Gendek, T.; Heimgartner, H. Helv. Chim. Acta 1998, 81, 1585. https://doi.org/10.1002/(SICI)1522-2675(19980909)81:9<1585::AID-HLCA1585>3.0.CO;2-N

15. Jasinski, M.; Mloston, G.; Mucha, P.; Linden, A.; Heimgartner, H. Helv. Chim. Acta 2007, 90, 1765. https://doi.org/10.1002/hlca.200790186

16. Antonova, M. M.; Baranov, V. V.; Nelyubina, Y. V.; Kravchenko, A. N. Chem. Heterocycl. Comp. 2014, 50, 1203.

https://doi.org/10.1007/s10593-014-1583-3

17. Cerecetto, H.; Gerpe, A.; Gonzalez, M.; Sainz, Y. F.; Piro, O. E.; Castellano, E. E. Synthesis 2004, 16, 2678. https://doi.org/10.1055/s-2004-831212

18. Alcazar, J.; Begtrup, M.; De La Hoz, A. J. Chem. Soc. 1995, 19, 2467. https://doi.org/10.1039/p19950002467

19. Mloston, G.; Celeda, M.; Prakash, G. S.; Olah, G. A.; Heimgartner, H. Helv. Chim. Acta 2000, 83, 728. https://doi.org/10.1002/(sici)1522-2675(20000412)83:4<728::aid-hlca728>3.0.co;2-b

20. Volkamer, K.; Zimmermann, H. W. Chem. Ber. 1969, 102, 4177. https://doi.org/10.1002/cber.19691021225

21. Kuruba, B. K.; Vasanthkumar, S.; Emmanuvel, L. Synth. Commun. 2016, 46, 799. https://doi.org/10.1080/00397911.2016.1176200

22. Rajkumar, R.; Kamaraj, A.; Krishnasamy, K. Journal of Taibah University for Science. 2015, 9, 498. https://doi.org/10.1016/j.jtusci.2014.12.001

23. Hasaninejad, A., Zare, A., Shekouhy, M.; Ameri-Rad, J. J. Comb. Chem. 2010, 12, 844. https://doi.org/10.1021/cc100097m

24. Sharma, S. D.; Hazarika, P.; Konwar, D. Tetrahedron Lett. 2008, 49, 2216. https://doi.org/10.1016/j.tetlet.2008.02.053

25. Gandhare, N. V.; Chaudhary, R.G.; Meshram, V. P.; Tanna, J. A.; Lade, S.; Gharpure, M. P.; Juneja, H. D. J. Chin. Adv. Mater. Soc. 2015, 3, 270. https://doi.org/10.1080/22243682.2015.1068134

26. Yugandar, S.; Konda, S.; Parameshwarappa, G.; Ila, H. J. Org. Chem. 2016, 81, 5606. https://doi.org/10.1021/acs.joc.6b00938

27. Mehrabi, H.; Alizadeh-Bami, F.; Ranjbar-Karimi, R. J. Iran. Chem. Soc. 2018, 15, 1961. https://doi.org/10.1007/s13738-018-1393-0

28. Mehrabi, H.; Najafian-Ashrafi, F.; Ranjbar-Karimi, R. Arkivoc 2018, (iii), 191. https://doi.org/10.24820/ark.5550190.p010.404 